\title{
Who is talking about \#Facelift on Instagram?
}

\author{
Cristina A. Salinas ${ }^{1} \cdot$ Doga Kuruoglu $^{2}(-) \cdot$ Horacio F. Mayer $^{3}\left([) \cdot\right.$ Tony C. Huang $^{2} \cdot$ Basel Sharaf $^{2,4}$
}

Received: 13 October 2021 / Accepted: 28 October 2021 / Published online: 2 December 2021

(c) The Author(s), under exclusive licence to Springer-Verlag GmbH Germany, part of Springer Nature 2021

\begin{abstract}
Background The internet has become a powerful tool for disseminating medical information. Social media platforms have gained popularity among medical professionals to connect with patients and promote their work and practices. We aimed to understand who is posting about facelift procedure on Instagram.

Methods Sprinklr, a social listening platform, was used to compile conversations that were posted on Instagram between January 1, 2021, and February 28, 2021, and contained hashtags \#facelift, \#necklift, \#plasticsurgery, \#plasticsurgerybeforeandafter, and \#platysmabands. English and Spanish language posts were analyzed for positive sentiment. Based on the number of likes, the top 100 English and Spanish posts were selected. Posts were categorized based on primary specialty of the poster as plastic surgeon, non-plastic surgeon, country of active clinical practice, board certification, and members of international plastic surgery societies.

Results A total of 5099 posts with a total of 11,918 mentions were collected. Of the top 100 English posts, the countries most represented were the USA (59\%), followed by Turkey (6\%). Physicians made up $48 \%$ of the top English posts, of whom $42 \%$ were US board-certified plastic surgeons. By contrast, of the top 100 Spanish posts, the countries most represented were Mexico (29\%) and Colombia (25\%). Physician posters constituted 78\%, of whom 38.5\% were plastic surgeons certified by their country of practice.

Conclusions Plastic surgeons have become more active in creating popular content related to facelift on Instagram over the past few years. However, a significant percentage of IG content on \#facelift, a surgical procedure, was made by non-physicians and non-plastic surgeons in 2021.
\end{abstract}

Level of evidence: Not gradable.

Keywords \#facelift $\cdot$ Social media $\cdot$ Hashtag $\cdot$ Aesthetic surgery $\cdot$ Plastic surgery $\cdot$ Instagram

Cristina A. Salinas and Doga Kuruoglu contributed equally and are co-first authors for this study.

Basel Sharaf

Sharaf.Basel@mayo.edu

1 Mayo Clinic Alix School of Medicine, Mayo Clinic, Rochester, MN, USA

2 Division of Plastic Surgery, Department of Surgery, Mayo Clinic, 200 1st Street SW, Rochester, MN, USA

3 Plastic Surgery Department, Hospital Italiano de Buenos Aires, University of Buenos Aires School of Medicine, Hospital Italiano de Buenos Aires University Institute, Buenos Aires, Argentina

4 Center for Aesthetic Medicine and Surgery (CAMS), Mayo Clinic, Rochester, MN, USA

\section{Introduction}

The internet has become a potent tool for disseminating medical information. Social media platforms have gained more popularity among medical professionals and patients for sharing information and promoting their practice [1]. Plastic surgery is a visual speciality and the use of images regarding plastic surgery procedures and results has increased significantly across different social media platforms. While those who practice aesthetic surgery are more likely to have a professional social media account [2], according to a study by Vardanian et al. [3], a majority of members of the American Society of Plastic Surgeons (ASPS) in 2013 had social media accounts.

The three social media platforms most used by plastic surgery patients include Facebook, YouTube and Instagram (IG), with the greatest interest shown in before-and-after photos, 
information on practices, and contests [4]. In 2017, most of plastic surgery posts on Twitter were made by the public instead of plastic surgeons [5]. Similarly in 2018, out of the top IG posts related to plastic surgery, only $17.8 \%$ were made by ASAPS eligible board-certified plastic surgeons. It was also found that some doctors who were not plastic surgeons were labeling themselves as "cosmetic surgeons." [6] Interestingly, it has also been found that the word "plastic" has negative associations, while the words "cosmetic, aesthetic, reconstruction" have positive associations on social media [7].

While social media platforms are becoming the most influential online method of selecting a plastic surgeon, even above Google [8, 9], a recent study by Fan et al. [10] found that $96 \%$ of the general public did not know what credentials a board-certified plastic surgeon should have. This confusion is amplified by social media accounts who falsely claim to be board-certified "cosmetic" or plastic surgeons, making it challenging for patients and the public to navigate social media pages and find qualified surgeons for the conditions and procedures they are seeking. Furthermore, it may be perceived that accounts with the greatest number of followers belong to more qualified or legitimate surgeons [11]. However, there is no evidence to support that the popularity of a social media account correlates with the highest level of care or surgical expertise. There are many paths to gain popularity on social media including sponsorships, agreements with companies to promote certain products and services, and becoming a social media influencer [11]. Moreover, not all followers on social media accounts are real. According to a recent study, nonphysicians had a higher number of fake bots compared with American board-certified surgeons [10]. These confounders further add to the difficulty in drawing parallels between surgical expertise and the popularity on social media.

A simple way to browse social media and find specific topics of interest is searching with a hashtag. In this study, we performed a qualitative analysis of the hashtag \#facelift among conversations on IG. \#Facelift was previously shown to be the most popular hashtag related to a specific procedure in plastic surgery [6]. We chose the two most common languages spoken in the United States to evaluate trends among both English and Spanish-speaking users. Accordingly, the top 100 English and Spanish languages posts were analyzed to assess who is posting about the topic of facelift and what differences are present in surgical training and representation of medical specialties of the accounts.

\section{Material and methods}

Sprinklr, a social listening platform that covers a range of social networks to better understand the customer experience, was used to compile IG conversations between January
1, 2021, and February 28, 2021. Only the conversations containing hashtags related to facelift including \#facelift, \#necklift, \#plasticsurgery, \#plasticsurgerybeforeandafter, and \#platysmabands were included and analyzed in this study. The posts were separated into English and Spanish languages and selected for positive sentiment. Posts not related to the medical field or facelifts were excluded. Based on the number of "likes," the top 100 English posts and 100 Spanish posts on IG were identified. These posts were then categorized based on the country of residence, gender, and profile of the IG account holder such as physician, patient, non-physician healthcare worker, plastic surgery practice, medical facility, make-up artist, skincare product, and other facilities (salons, recovery homes, medical tourism). The posts made by physicians were further categorized based on their primary specialty: plastic surgery, oculofacial plastic surgery, ear nose and throat (ENT), oral and maxillofacial surgery, cosmetic surgery, general surgery, venous and lymphatic medicine, general practitioner, and orthodontist. The posts by physicians were sorted by country of active clinical practice and board certification that the physician holds. Board certification is voluntarily obtained and can only be done after completing the required training in medical school and an accredited residency program and passing a written and oral examinations covering all plastic surgery procedures. Membership status of physicians in plastic surgery societies including American Society of Plastic Surgeons (ASPS), International Society of Aesthetic Plastic Surgery (ISAPS), and Ibero-Latin-American Federation of Plastic Surgery (FILACP) was noted.

\section{Results}

A total of 5,099 posts with a total of 11,918 mentions were collected. Of the top 100 English posts selected, the countries most represented were the USA (59\%), Turkey (6\%), and Australia and Canada (each 5\%) (Fig. 1). Forty-eight percent of the posts were made by physicians (Fig. 2), $42 \%$ of whom were US board-certified plastic surgeons, $25 \%$ international plastic surgeons, and $17 \%$ American boardcertified ENT surgeons. Other board certifications included cosmetic surgery, ophthalmic plastic and reconstructive surgery, oral and maxillofacial surgery, and venous and lymphatic medicine (Fig. 2). Fifteen of the physicians (31\%) were members of ASPS, and 4 of the physicians (8\%) were members of ISAPS. Of the top 100 posts, $12 \%$ were by plastic surgery practices. Forty-six percent of the total 100 English posts were made by male users, and $21 \%$ were by female users. The post with the most likes was by a US board-certified ENT surgeon. The post with the second highest number of likes was by a US board-certified plastic surgeon. 
Fig. 1 Number of English language posts according to country of origin
Fig. 2 The Top 100 English language posts by posting account credential and specialty
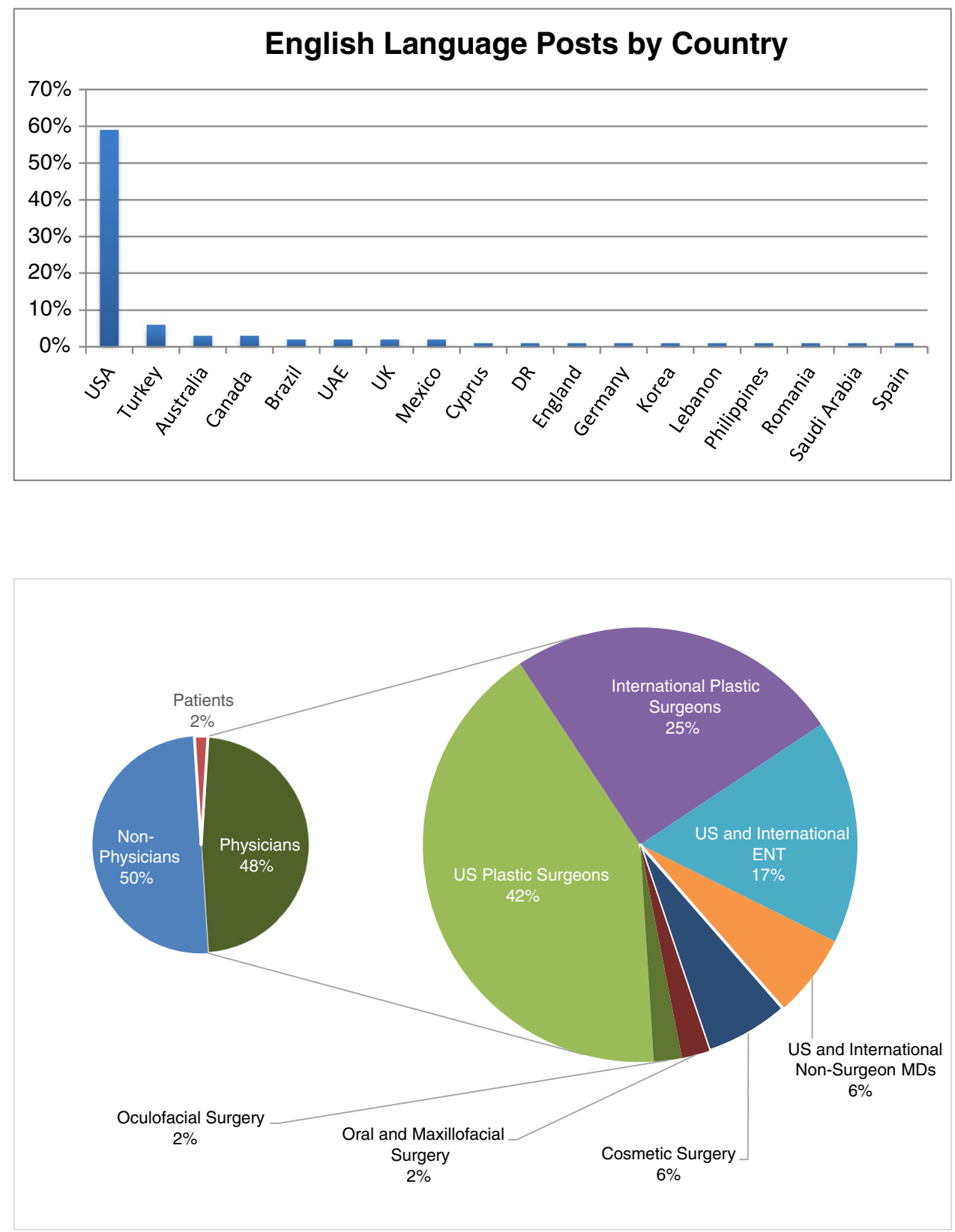

Of the top 100 Spanish posts, the countries most represented were Mexico (29\%), Colombia (25\%), and Venezuela (10\%) (Fig. 3). Seventy-eight percent of the posts came from physicians, $79.5 \%$ of whom were plastic surgeons (Fig. 4). Only $49 \%$ of these plastic surgeons were certified by the country they currently practice in. Of the physician posts, $6.4 \%$ were general surgeons and $5.1 \%$ were ENT physicians (Fig. 4). Fourteen of the physicians (18\%) were either members of ASPS or ISAPS, and 31 (40\%) were members of FILACP. Of the total 100 Spanish posts, $62 \%$ were by male users, and $24 \%$ were by female users. Six percent of the top $100 \mathrm{Spanish}$ posts came from certified plastic surgery practices. The post with the most likes was made by a plastic surgeon certified in Mexico, followed by a post by a plastic surgeon certified in Bolivia.

Ninety-five percent of the mentions were associated with a positive sentiment. The top themes of discussion in these top posts were plastic surgery, skin care, face, face lifting, skin tightening, and self-care.

\section{Discussion}

Over the past decade, social media has become an important platform for disseminating information that influences all aspects of our lives, including hosting news coverage and 
Fig. 3 Number of Spanish language posts according to country of origin
Fig. 4 The Top 100 Spanish language posts by posting account credential and specialty
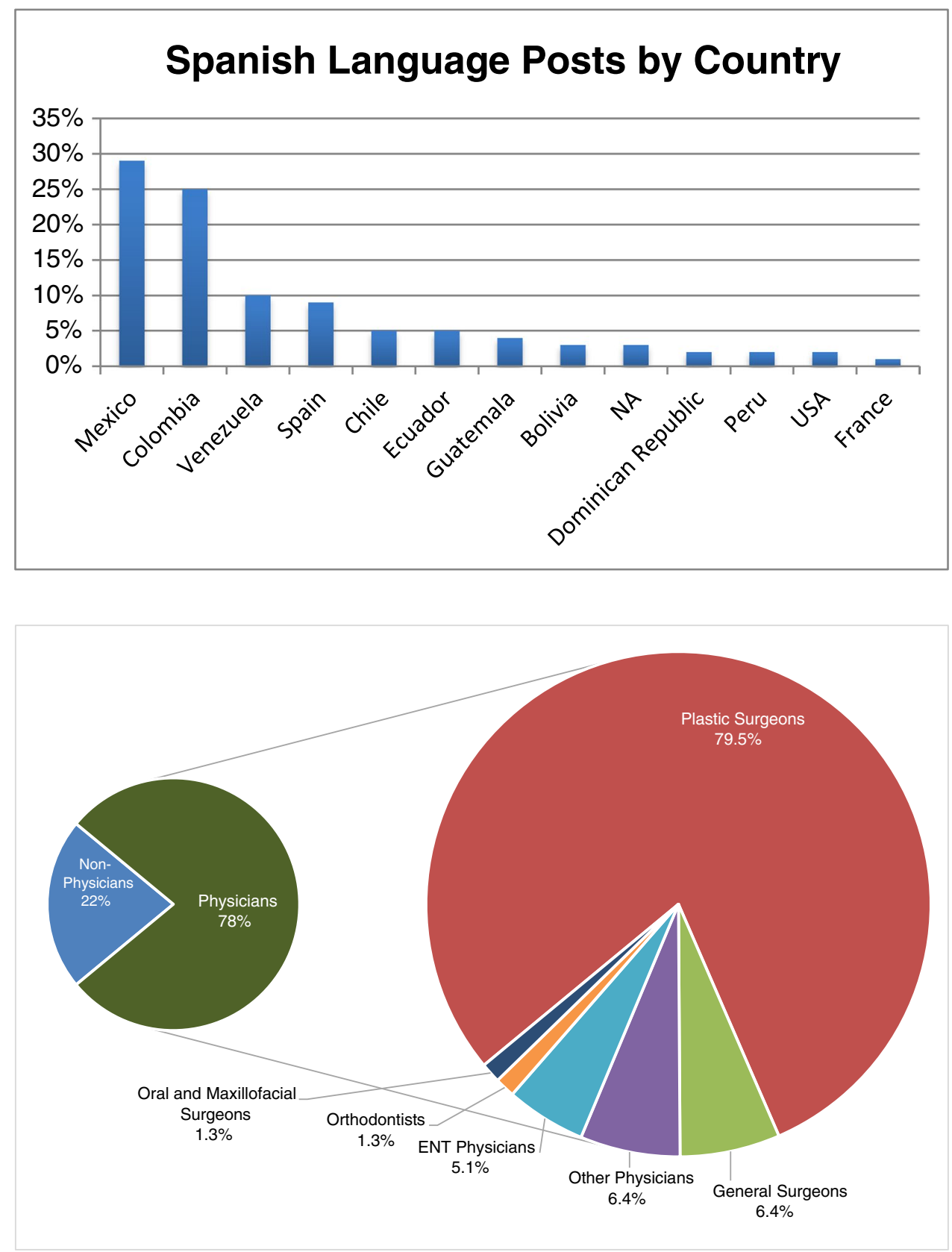

political discussions, advertising products and services, and promoting cosmetic procedures to patients. Recent studies evaluated the presence of plastic surgery in these platforms $[6,7,10-12]$. Some studies utilized general hashtags in plastic surgery, such as \#Plasticsurgery and \#aestheticsurgery, posted on popular platforms such as Twitter and Instagram $[5,7,12]$. Despite the explosive emergence of social media in aesthetic surgery over the past 5 years, published literature regarding specific plastic surgery procedures trends in social media is still limited. In this study, we aimed to analyze the social media content related to "facelift" that was posted on IG over 2 months by using Sprinklr (New York, NY), an advanced social media analytics software.
In our study, the "top" 100 English language posts related to facelift were mostly from the United States (59\%), followed by Turkey (6\%), Canada (5\%), and Australia (5\%). Of all the posts analyzed, $48 \%$ were made by physicians, demonstrating that the other half of the posts were made by non-physician IG posters. Among the physician posters, US board-certified plastics surgeons constituted the most represented group (42\%) in the top 100 facelift IG posts in English. In 2017, Dorfman et al. [6] analyzed the plastic surgery-related content on Instagram, reviewing 1,789,270 posts. The authors used a total of 21 hashtags that were related to popular plastic surgery procedures to identify what type of plastic surgery-related content was being posted, who 
was posting this content, and which hashtags were being used. The most popular hashtag was \#plasticsurgery (29\%), followed by \#facelift (12.5\%) and \#rhinoplasty (7.7\%). When the top 9 posts of the 21 hashtags were further analyzed, the authors reported that $42 \%$ of the posts were from surgeons outside the US, with Turkey being the most prominent among them (25\%). In their study, physicians were responsible for $77 \%$ of the posts, which has decreased to $48 \%$ in our study. However, the authors also noted that US board-certified plastic surgeons were underrepresented among physicians in their cohort, unlike our study findings. When we reviewed the top 100 posts in the Spanish language, we saw that most were from Mexico (29\%), followed by Colombia (25\%). Boardcertification is a safeguard to ensuring safe and ethical plastic surgery practices by maintaining high standards for education, assessment of knowledge, and continuous certification of plastic surgeons [13]. While board-certification in plastic surgery has well delineated paths in the USA and Canada, the training and certification requirements can vary in Central and South American Countries. As recently reported by Mayer et al. [14] plastic surgery training in Latin American countries is very diverse in terms of requirements, programs content and duration, evaluation and certifying authorities. In these countries, a plastic surgery degree can be obtained after completing either a residency training program at public or private hospitals or a post-graduate course taught at universities or scientific societies. Therefore, a board certification can be awarded by the ministry of health, university, scientific society, or medical council. With regard to contents, although most programs cover the relevant fields of the specialty, not every program offers hands-on training in aesthetic surgery; an important prerequisite to ensure adequate experience prior to independent practice after graduation. The percentage of plastic surgeons associated with their respective national plastic surgery societies is also quite variable in this part of the world, ranging from 100\% in Panama to $40 \%$ in Argentina [14].

The majority of the top 100 IG posts were made by physicians (78\%), of which plastic surgeons were the most represented group (79.5\%). Of the plastic surgeons who made the top 100 IG posts in Spanish, 49\% were plastic surgeons certified by the country in which they currently practice. These findings demonstrate that both, English-speaking and Spanish-speaking board-certified plastic surgeons have become more active and popular users of Instagram among physicians over the last few years. This is indicative of an increased awareness among the plastic surgery community of the importance of social media in attracting patients and curating an experienced and reliable image for their practice.

In a 2016 study that analyzed 2880 "tweets" containing the words "plastic surgery," Branford et al. [5] reported that the social media posts about plastic surgery were dominated by the public, making up $70.6 \%$ of the tweets compared to only $6 \%$ by plastic surgeons. Similarly, in our study, nonphysician IG posts represented 52\% of the top 100 IG English posts related to \#facelift.

In this study, we selected Instagram among all other social media platforms to analyze a large volume of faceliftrelated topic given its increased popularity as a podium for plastic and cosmetic surgery content. In a recent study by Mullens et al. [12], the authors compared the \#plasticsurgery posts on Instagram versus Twitter over 30-consecutive days in 2020. They reported that IG had a significantly higher volume of \#plasticsurgery posts than Twitter within that period. In addition, they noted that post engagements were significantly higher on IG compared to Twitter.

It is also worth to discuss the presence of both non-plastic surgeons and non-physicians as IG posters addressing the historically surgical topic of facelift. Our study confirms the predominance by non-physicians and non-plastic surgeons on popular facelift-related content on IG. Non-physicians constituted $52 \%$ and non-plastic surgeons made $68 \%$ of the top 100 IG posts in English. This trend was different among the posts in Spanish, where only 22\% of the top 100 posts were authored by non-physicians and $38 \%$ of the top 100 were by non-plastic surgeons. Previous literature had varying data regarding plastic surgery content and poster profiles on IG. In a study by Mullens et al. [12] in 2020, it was reported that $45 \%$ of the plastic surgery-related posts on IG were created by non-plastic surgeons and $8.5 \%$ by other physicians or health professionals. In the study by Dorfman et al. [6], non-physician authors constituted 5.5\% of the top \#plasticsurgery posts; a lower rate than the $52 \%$ in our study in which we exclusively analyzed facelift-related posts. The differences in the rates between our study and these two studies by Dorfman et al. [6] and Mullens et al. [12] may be explained by the different study designs, the topics of search with plastic surgery being more encompassing than face-lift, as well as the growing trend of IG use among non-physicians and non-plastic surgeons to communicate to the public about cosmetic surgery.

It was shown that the coronavirus disease 2019 (COVID19) pandemic has impacted the public's interest in aesthetic plastic surgery procedures, which also reflected to the use of social media in this regard $[15,16]$. In a recent study, Thawanyarat et al. [15] investigated the trends in search terms related to aesthetic surgery procedures following the COVID-19 pandemic, by gathering the search data between January 2015 and March 2021 via Google Trends (Google, Mountain View, California, USA). The authors reported that the terms including platysmaplasty, blepharoplasty, Botox, brachioplasty, breast implant removal, breast reduction, brow lift, buccal fat removal, hair transplantation, lip augmentation, mentoplasty, otoplasty, rhinoplasty, and thighplasty had statistically significant increases in search volumes after the chosen infection point, February 2020. These findings were associated with the increased use of 
video conferencing, leading to a demand towards facial aesthetic procedures $[15,16]$.

Because non-physicians and non-plastic surgeons still make up most of the IG posts regarding facelift and other plastic surgery procedures, there is the concern that potential patients are not obtaining accurate information on social media platforms and instead are being drawn to individuals and practices that have not received the required training to offer safe and high-quality care. It is becoming more important than ever for plastic surgeons to create a strong social media presence that reaches beyond their immediate community to educate the public on the risks and benefits of the procedure they offer, such as facelifts, and make it easy for patients to find properly trained and board-certified surgeons among the various other physicians posting on these same procedures.

This study has some limitations including the use of one social media analytics software to analyze past IG posts and the potential for selection bias. Furthermore, posts in languages other than English and Spanish were not included this study which limits the generalizability of our results to other regions of the world, such as Asia. Also, some IG posts may have been missed in the search by Sprinklr as we limited our search terms to lay person lingo or common words used in social media. However, it was emphasized by a recent study that lay person terminology is more widely used in IG than proper medical terminology (i.e., \#facelift versus \#rhytidectomy, \#tummytuck versus \#abdominoplasty) [6].

\section{Conclusions}

Plastic surgeons are becoming more active in the conversation on facial aesthetic surgery. However, non-physicians and non-plastic surgeons continue to make up a significant portion of the content on \#facelift, a surgical procedure. It is important for both academic and private practice plastic surgeons to continue to share reliable content on social media pertaining to aesthetic facial surgery to increase awareness and educate the public.

\section{Declarations}

Conflict of interest Author Dr. Horacio F. Mayer is the Editor-in-Chief of European Journal of Plastic Surgery. The authors have no other relevant financial or non-financial interests to disclose.

Ethical approval For this kind of article, formal consent from a local ethics committee is not required.

Patient consent Not applicable.

\section{References}

1. Teven CM, Park JE, Song DH (2017) Social Media and Consent: Are Patients Adequately Informed? Plast Reconstr Surg 140(5):770e-771e. https://doi.org/10.1097/PRS.0000000000003805

2. Economides JM, Fan KL, Pittman TA (2019) An Analysis of Plastic Surgeons' Social Media Use and Perceptions. Aesthet Surg J 39(7):794-802. https://doi.org/10.1093/asj/sjy209

3. Vardanian AJ, Kusnezov N, Im DD, Lee JC, Jarrahy R (2013) Social media use and impact on plastic surgery practice. Plast Reconstr Surg 131(5):1184-1193. https://doi.org/10.1097/PRS.0b013e3182 $87 \mathrm{a} 072$

4. Sorice SC, Li AY, Gilstrap J, Canales FL, Furnas HJ (2017) Social media and the plastic surgery patient. Plast Reconstr Surg 140(5):1047-1056. https://doi.org/10.1097/PRS.0000000000 003769

5. Branford OA, Kamali P, Rohrich RJ et al (2016) \#PlasticSurgery. In: Plastic and Reconstructive Surgery. Plast Reconstr Surg 138:1354 1365. https://doi.org/10.1097/PRS.0000000000002814

6. Dorfman RG, Vaca EE, Mahmood E, Fine NA, Schierle CF (2018) Plastic Surgery-Related Hashtag Utilization on Instagram: Implications for Education and Marketing. Aesthet Surg J 38(3):332-338. https://doi.org/10.1093/asj/sjx120

7. Chopan M, Sayadi L, Clark EM, Maguire K (2019) Plastic Surgery and Social Media: Examining Perceptions. Plast Reconstr Surg 143(4):1259-1265. https://doi.org/10.1097/PRS.0000000000 005445

8. Montemurro P, Cheema M, Hedén P (2018) Patients' and Surgeons' Perceptions of Social Media's Role in the Decision Making for Primary Aesthetic Breast Augmentation. Aesthet Surg J 38(10):10781084. https://doi.org/10.1093/asj/sjy021

9. Gould DJ, Grant Stevens W, Nazarian S (2017) A Primer on Social Media for Plastic Surgeons: What Do I Need to Know About Social Media and How Can It Help My Practice? Aesthet Surg J 37(5):614 619. https://doi.org/10.1093/asj/sjw246

10. Fan KL, Graziano F, Economides JM, Black CK, Song DH (2019) The Public's Preferences on Plastic Surgery Social Media Engagement and Professionalism: Demystifying the Impact of Demographics. Plast Reconstr Surg 143(2):619-630. https://doi.org/10.1097/ PRS.0000000000005205

11. Gupta N, Dorfman R, Saadat S, Roostaeian J (2020) The Plastic Surgery Social Media Influencer: Ethical Considerations and a Literature Review. Aesthet Surg J 40(6):691-699. https://doi.org/10. 1093/ASJ/SJZ329

12. Mullens CL, Hardy KM, Hernandez JA et al (2020) \#PlasticSurgery: A Comparative Deep Dive Analysis into Social Media and Plastic Surgery. Plast Reconstr Surg 146(2):413-422. https://doi.org/10. 1097/PRS.0000000000007001

13. The American Board of Plastic Surgery. ABPlasticsurgery.org. https://www.abplasticsurgery.org/about-us/mission-statement/. Accessed October 11, 2021

14. Mayer HF, Jacobo OM, Grattarola G (2021) Plastic surgery training in Ibero-Latin America: A cross-sectional survey study. J Plast Reconstr Aesthet Surg 74(7):1633-1701. https://doi.org/10.1016/j. bjps.2020.12.097

15. Thawanyarat K, Francis S, Kim T, Arquette C, Morrison S, Nazerali R. The Zoom Effect: A Google Trends Analysis. Aesthetic Surg J. Published online September 28, 2021. https://doi.org/10.1093/asj/ sjab347

16. Gülbitti HA, Waziri S, van der Lei B (2021) From Some-tox to More-tox during the COVID-19 Pandemic. Plast Reconstr Surg; Publish Ah(4). https://doi.org/10.1097/prs.0000000000008366

Publisher's note Springer Nature remains neutral with regard to jurisdictional claims in published maps and institutional affiliations. 